\title{
12 \\ DAS FRONTEIRAS INSTITUCIONAIS À MOBILIZAÇÃO SOCIAL: INTERVENÇÕES NO PROCESSO DE DOAÇÃO-TRANSPLANTE
}

\author{
From instututional borders to social mobilization: Interventions in the donation and \\ organ transplantation process
}

\author{
Heloisa Rosário Furtado Oliveira Lima', Francisca Georgina Macedo de Sousa², Elza Lima da Silva²
}

\begin{abstract}
RESUMO
Objetivo: Construir a partir da ativa participação dos atores envolvidos no processo de doação, captação e transplante de órgãos, medidas inovadoras visando aumento das notificações, doações e transplantes no estado do Maranhão. Métodos: Pesquisa exploratória, descritiva com abordagem qualitativa guiada pelo modelo da Pesquisa Convergente Assistencial. Os dados foram coletados pelos seguintes recursos: rodas de conversa, grupos focais, encontros individuais e cotidiano das práticas que envolvem o processo de doação de órgãos e tecidos. Resultados: Apresentados sob três dimensões: institucional, técnica-profissional e mobilização social. Conclusão: As intervenções definiram-se como medidas inovadoras com repercurssões positivas refletindo-se no aumento de notificações de morte encefálica, doação e transplantes de órgãos, fazendo com que o estado do Maranhão saísse da $18^{a}$ para a $16^{a}$ posição no ranking nacional.
\end{abstract}

Descritores: Doação de Órgãos; Doação de Tecidos; Transplante.

\section{Institution:}

${ }^{1}$ Comissão Intra-hospitalar de Doação de Órgãos e Tecidos para Transplante do Hospital Municipal Djalma Marques e Hospital Universitário da UFMA. São Luís, Maranhão, Brasil.

${ }^{2}$ Curso de Graduação em Enfermagem da Universidade Federal do Maranhão da UFMA. São Luís, Maranhão, Brasil.

\section{Correspondence:}

Heloisa Rosário Furtado Oliveira Lima

Rua dos Bicudos, Cond.Acapulco Residence, Bloco 2, Apto.1101, CEP 65075-838, São Luís/MA, Brasil.

Tel.: (98) 98119-1312

E-mail: heloisalima66@gmail.com

\section{INTRODUÇÃO}

O primeiro transplante renal no Brasil ocorreu em 1964, e em 1965 foi desenvolvido o primeiro programa de transplante do país. Em 1968 foi realizado o primeiro transplante de coração, amparado somente pelo critério de morte encefálica (ME). Essa condição impulsionou a comunidade científica brasileira a elaborar uma legislação para garantir a realização dos próximos transplantes de órgãos. Tal legislação tinha como finalidade resguardar os direitos dos indivíduos envolvidos e a garantia da gratuidade da cessão dos órgãos e tecidos. ${ }^{1}$

Os transplantes no Brasil foram divididos arbitrariamente, em três fases, de acordo com os seguintes critérios: conhecimento médico, estruturas hospitalares, organização do sistema tanto de procura quanto de alocação e controle e financiamento. ${ }^{2}$ 
A primeira fase ou heroica ocorreu de 1964 a meados de 1987, caracterizada pela falta de remuneração e estrutura, conhecimento limitado e sem controle, onde um médico da equipe de transplante renal visitava ou telefonava para os serviços de terapia intensiva, solicitando ser comunicado em caso de ME, para que se realizasse a entrevista familiar. ${ }^{2}$

A segunda fase ou romântica foi do final de 1987 até 1997, quando era evidenciado melhor conhecimento da imunologia dos transplantes, hospitais mais estruturados, iniciada a regulamentação, criação de organizações de procura/alocação de órgãos e remuneração limitada. ${ }^{2}$

A terceira fase ou profissional iniciou-se em 1997 com a Lei no. $9434^{3}$ e o Decreto Lei no. $2268{ }^{4}$ do Ministério da Saúde (MS) que a regulamentou, mudando a forma de consentimento, criando o Sistema Nacional de Transplante (SNT), órgão central com sede em Brasília e as centrais estaduais de transplante, denominadas Centrais de Notificação, Captação e Distribuição de Órgãos (CNCDO) ou Centrais Estaduais de Transplante (CETx,) que englobam a logística do processo de doação e transplante no seu âmbito, além da responsabilidade pela política de transplante. ${ }^{2}$ Nessa fase, todo o controle do transplante no país passou a ser de responsabilidade do governo federal e foram tomadas medidas nas quatro áreas consideradas os pilares de transplantes: ${ }^{2}$

a) Financiamento: Em 1998, foi estabelecido um fundo federal (FAEC - Fundo de Ações Estratégicas e Compensação) para financiamento dos transplantes, imunossupressores, todas as atividades relacionadas à doação e pós-transplantes;

b) Legislação: Uso do consentimento presumido e da decisão obrigatória, proibição do comércio, restrição ao doador vivo não relacionado (autorização judicial), comunicação prévia ao Ministério Público de todos os transplantes de órgãos com doador vivo, critérios para credenciamento de centros e equipes com renovação a cada dois anos e critérios nacionais de alocação, com listas de espera estaduais, com distribuição pelas centrais estaduais e foram estabelecidas as penas em caso de não cumprimento da lei;

c) Organização: Modelo baseado no espanhol de forma incompleta, pois foram criadas as organizações nacional e estaduais, mas que não valorizavam as organizações hospitalares (Coordenações hospitalares de transplantes);

d) Educação: Foram iniciados cursos de formação de coordenadores hospitalares de transplante entre 1999 e 2004 em 18 estados e no Distrito Federal pelo MS em parceria com a Universidade de Brasília (UnB) e a Fundação Nacional de Saúde (FUNASA).
A legislação atribuiu a autoridade para determinação de ME ao Conselho Federal de Medicina (CFM), e em 1997 foi publicada uma resolução para o diagnóstico de ME. Os testes clínicos devem ser realizados por dois médicos não pertencentes às equipes de remoção ou transplante, sendo obrigatórios dois testes com intervalo mínimo entre eles, de acordo com a idade e um teste de imagem. Entretanto, o decreto-lei exige que um dos médicos seja especialista em neurologia ou neurocirurgia. ${ }^{4}$

Em 2000 e 2005, por meio de portarias foram criadas:

a) Central Nacional de Notificação, Captação e Distribuição de Órgãos (CNNCDO) ou Central Nacional de Transplante (CNT), com o objetivo de gerenciar lista única nacional, articular a distribuição de órgãos entre os estados, priorizar as urgências e possibilitar o transporte em parcerias com companhias aéreas, ${ }^{2,5}$

b) Comissão Intra-Hospitalar de Transplante (CIT) ou Comissão Intra-Hospitalar de Doação de Órgãos e Tecidos para Transplante (CIHDOTT) ou Coordenação Hospitalar de Transplante (CHTx), cuja portaria determina que todos os hospitais com potencial de doação devem criar essa comissão para viabilizar a doação na instituição. Porém, por não ter sido definida a forma de pagamento e financiamento, na maioria das instituições, as comissões somente foram criadas no papel e nunca atuaram..$^{2,6}$

Em 2009, a portaria no. 2600 GM/MS publicou o regulamento técnico do transplante, onde foi prevista a formação de Organizações de Procura de Órgãos (OPO), uma para cada dois milhões de habitantes, financiada pelo governo federal. De acordo com essa portaria, as funções do SNT serão exercidas pelo MS, por intermédio da Coordenação Geral do SNT (CGSNT), órgão com atuação no âmbito federal, que tem como objetivos harmonizar as políticas nacionais do SNT e articular o sistema com os outros órgãos do MS, do Departamento de Atenção Especializada (DAE) e da Secretaria de Atenção à Saúde (SAS). ${ }^{7}$

Atualmente, o MS mantém uma rede integrada composta por 33 CNCDOs, incluindo as cinco Regionais de Minas Gerais e duas de São Paulo; 12 Câmaras Técnicas Nacionais (CTNs), um Grupo de Assessoramento Estratégico (GAE), 500 Centros de Transplantes (hospitais), 70 Organizações de Procura de Órgãos (OPOs) e 628 CIHDOTTs em 26 Estados e no Distrito Federal. $^{8}$

As CNTs são grupos para assessoria técnica da CGSNT com a finalidade de estudar e sugerir ao MS a formulação, revisão, atualização e aperfeiçoamento das normas relativas ao transplante. O GAE tem como atribuições: 
elaborar diretrizes para política de transplantes e enxertos, propor temas de regulação complementar, identificar indicadores de qualidade para as atividades de doação e transplantes, analisar relatórios com dados de atividades do SNT e emitir parecer quando solicitado pela CGSNT. ${ }^{7}$

Cabe à Secretaria Estadual de Saúde a criação, no âmbito da CNCDO das Câmaras Técnicas Estaduais de Transplante (CTEs) formada por profissionais nomeados para estabelecer os critérios de cadastramento de candidatos a receptores de diferentes órgãos e os critérios de urgência, fundamentados nos critérios mínimos preconizados na legislação, além de analisar, avaliar e decidir nos casos de solicitação de inscrição de pacientes indicados para transplantes, mas que não se enquadram nos critérios preconizados pelo MS. Enquanto as CTEs não forem criadas, as solicitações de inscrição nos cadastros técnicos de receptores que dependem de aprovação das mesmas deverão ser apreciadas pelos órgãos técnicos da Coordenação da CNCDO. ${ }^{2}$

O Maranhão iniciou as atividades de transplante em 2000, com realização de transplante de córnea e rim. Entretanto, o crescimento ainda é lento, colocando o Estado numa condição inferior quando comparado aos demais estados, incluindo os do Nordeste, que apresentam crescimento na doação e número de transplantes. ${ }^{9}$

Essa situação, impulsionou-nos a buscar estratégias, visando as potencialidades do estado para elevar as doações por meio do questionamento: quais instrumentos, recursos e estratégias podem ser utilizados para aumentar os doadores possíveis e efetivos de órgãos e tecidos no Maranhão?

O foco da investigação foi modificar o diagnóstico situacional da doação de órgãos e transplante no estado do Maranhão, uma vez que o conhecimento dos obstáculos e das resistências ao processo viabilizou a construção e a elaboração de estratégias e recursos de intervenção, promovendo melhores resultados no processo de doação e transplante de órgãos e tecidos.

O objetivo da pesquisa foi construir a partir da ativa participação dos atores envolvidos no processo de doação, captação e transplante de órgãos medidas inovadoras visando o aumento das notificações, doações e transplantes no estado do Maranhão.

\section{MÉTODOS}

Pesquisa exploratória, descritiva de natureza qualitativa, guiada pela Pesquisa Convergente Assistencial (PCA), desenho metodológico que viabiliza ao profissional da saúde envolver-se concomitantemente com a assistência e pesquisa. A PCA demanda estreita relação da pesquisa com a prática assistencial com o propósito de descobrir alternativas para resolver ou minimizar problemas, realizar mudanças e/ou introduzir inovações no contexto da prática em que ocorre a investigação, e deve ser produzida na mesma área e tempo de determinada prática, onde o pesquisador desenvolve simultaneamente pesquisa e prática de saúde com a intenção de promover mudanças qualificadoras daquela assistência. ${ }^{10}$

Os participantes da pesquisa foram definidos a partir do enunciado de Trentini e Paim ${ }^{10}$ de que a PCA é um método de pesquisa que consiste em elucidar problemas sociais e técnicos e encaminhar estratégias para solução a curto, médio e longo prazos, dependendo do problema evidenciado. Para tanto, são participantes da pesquisa grupos em que se encontram reunidos pesquisadores, membros da situação-problema e outros atores e parceiros interessados na resolução dos problemas, ou, pelo menos, no avanço a ser dado para que sejam formuladas adequadas responsabilidades sociais, educacionais, técnicas e/ou políticas.

Nessa metodologia, os participantes têm papel diferente quando comparado aos das demais modalidades de pesquisa, pois aqui, estes configuram-se como sujeitos partícipes em interação com peritos investigadores. Nesse sentido, forma-se uma equipe de pesquisa integrada unindo pesquisadores e agentes de mudanças, em um processo dinâmico, cooperativo, participativo e interativo entre criadores do projeto de pesquisa e os representativos da realidade investigada. Os participantes são, portanto, construtores não de uma pesquisa, mas de um projeto de vida. ${ }^{10}$

A partir dos pressupostos da PCA, participaram da pesquisa 50 profissionais inseridos nas Comissões Intra-hospitalares de Doação de Órgãos e Tecidos para Transplantes (CIHDOTTs), Central de Notificação, Captação e Distribuição de Órgãos (CNCDO) do Estado, Banco de Olhos (BO) do Hospital Universitário da Universidade Federal do Maranhão (HU-UFMA), Secretaria Municipal de Saúde, Promotoria de Justiça do Estado, Superitendência de Controle, Avaliação, Auditoria e Regulação de Leitos do Município.

Os dados foram coletados com a utilização dos seguintes recursos: rodas de conversa, grupo focal e encontros individuais visando identificar problemas, dificuldades, elencar intervenções e estabelecer consensos.

As condições éticas para realização da pesquisa foram aprovadas sob parecer no. 747.481/ 2014. A pesquisa teve apoio financeiro da Fundação de Amparo à Pesquisa e Desenvolvimento Científico do Maranhão (FAPEMA). 


\section{RESULTADOS}

Os resultados, compreendidos aqui como produtos da pesquisa por meio do método da PCA serão apresentados sob três dimensões: institucional, técnico-profissional e mobilização social fundamentados nas discussões dos recursos de coleta de dados, como abaixo descritas:

\section{a) Dimensão Técnico-Institucional}

Foram constituídas duas CIHDOTTs no município de São Luís, capital do estado do Maranhão/Brasil;

Dois hospitais do município de São Luís ampliaram o quadro de pessoal para atuarem nos serviços de doação e transplante de órgãos, perfazendo um total de 17 profissionais;

Realizada capacitação dos profissionais de saúde e de Coordenadores das áreas críticas de hospital de urgência e emergência, Banco de Olhos, Laboratório de Estudos Genômicos e de Histocompatibilidade, CIHDOTT e CNCDO direcionada ao manejo do potencial doador (PD);

Realização da Semana do Doador de Órgãos;

Realização de capactitação dos profissionais do Serviço de Atendimento Móvel de Urgência (SAMU) visando assegurar suporte para o processo de doaçãotransplante;

Realização do $1^{\circ}$ Ciclo de Palestras da CIHDOTT abordando temas sobre doação de órgãos e tecidos;

Construção e publicação de ferramenta pedagógica "Guia Prático para o Manejo de Morte Encefálica", com a finalidade de apoiar as decisões dos profissionais nas etapas do processo de identificação, avaliação e manutenção do paciente em ME;

$\mathrm{Na}$ institucionalização da cobrança dos procedimentos de doação e transplante identificou-se que os hospitais localizados no município de São Luís, apesar de realizarem procedimentos relativos à doação e transplante, não faziam a cobrança dos mesmos. Para minimizar o problema, foi enviado ofício esclarecendo como proceder a cobrança dos procedimentos, anexando a Tabela de Procedimentos Realizados pela Equipe da CIHDOTT. O produto dessa intervenção foi a qualificação da cobrança dos procedimentos;

Definição do espaço físico específico para a entrevista familiar;

Capacitação e acompanhamento técnico aos profissionais para a entrevista familiar;

Disponibilização de leito na terapia intensiva aos pacientes do centro cirúgico com diagnóstico de ME, possibilitando a visualização desses pacientes como críticos;
Realização da abordagem contínua junto aos profissionais de saúde, visando qualificar o protocolo de ME e agilidade na finalização do mesmo;

Aquisições de equipamentos (oxímetro, computador, HD externo, impressora) para a CIHDOTT;

Distribuição do Guia Prático para Manejo da Morte Encefálica nas Unidades de Saúde de ProntoAtendimento (UPAs), Hospitais de Urgência e SAMU de São Luís e São José de Ribamar;

Divulgação para a equipe médica dos hospitais de Urgência e Emergência a decisão do MS que define como legal e ética a suspensão de suportes terapêuticos quando diagnosticada ME em não doador; ${ }^{11}$

Confecção de adesivo com a descrição dos exames necessários para avaliação do PD;

Instituição da realização do exame complementar, conforme determina a Resolução no 1480/97, do Conselho Federal de Medicina (CFM) para caracterizar e documentar ausência de fluxo vascular ou de atividades eletrofisiológica ou metabólica; ${ }^{4}$

Na supervisão para manejo do PD foram estabelecidas as estratégias: acompanhamento in loco dos casos de ME pelo médico da CNCDO e adoção do Guia para manejo do paciente em ME, o qual define o valor do sódio, a temperatura corporal e a vida média das drogas sedativas;

Divulgação aos profissionais de saúde das principais legislações do processo doação-transplante, entre elas: lei que dispõe sobre remoção, tecidos e partes do corpo humano para fins de transplante, tratamento e dá outras providências; os critérios de $\mathrm{ME;}$; suspensão de suportes terapêuticos; ${ }^{11}$ e aprovação do Regulamento Técnico do Sistema Nacional de Transplante; ${ }^{7}$

Instituição da abordagem familiar em tempo hábil (seis horas pós-óbito), com agilidade na liberação da Declaração de Óbito, viabilização da entrega de documentos do paciente na admissão e solicitado apoio da Comissão de Óbito para correções das informações nas declarações;

Esclarecimento aos médicos para não colocar na Declaração de Óbito a causa básica "sepse", quando o paciente tiver apresentado boa resposta clínica à antibioticoterapia ou ocorrido tratamento efetivo do quadro séptico, pois esse diagnóstico inviabiliza a entrevista familiar para doação. Segundo a Portaria no. 2600/2009, somente a sepse refratária (infecção bacteriana generalizada, com hipotensão e consequentemente sofrimento de órgãos como rins, fígado e pulmões, que não responde aos medicamentos) é considerada critério absoluto de exclusão de doador de órgãos, tecidos, células ou partes do corpo humano; ${ }^{7}$ 
Realização dos esclarecimentos aos médicos sobre o primeiro exame clínico para diagnóstico de ME. Rotineiramente, esses profissionais argumentavam que o primeiro exame deveria ser realizado pelo neurologista. Porém, aos poucos eles foram convencidos de que o ideal seria acelerar o processo, sendo determinado que o médico que identificasse a ausência dos elementos do exame neurológico (coma aperceptivo, pupilas fixas e arreativas, ausência de reflexo córneo-palpebral, reflexo óculo-cefálico, resposta às provas calóricas, reflexo de tosse e apneia) iniciaria a abertura do protocolo de ME; Estimulada a abertura de protocolo de ME nas UPAs, com duas propostas de intervenções entre CNCDO e Secretaria Estadual de Saúde: regulação dos pacientes em ME para o hospital estadual e contratação de neurologista para realizar exame complementar de confirmação da ME. Atualmente, o protocolo de ME prevê que o primeiro exame clínico deve ser realizado pelo médico assistencial e o doppler transcraniano por neurologista contratado pelo estado;

Utilização das redes sociais pelos profissionais da CNCDO e CIHDOTT permitiu interação e agilização das notificações de óbitos e ME.

É comum os profissionais de saúde que realizam busca ativa de órgãos e tecidos serem recebidos nos serviços com apelidos desrespeitosos e de baixo calão, caracterizados como assédio moral. Como intervenção, foram definidas as seguintes estratégias: promover educação permanente sobre o processo de doação de órgãos e tecidos; denunciar os casos de assédio moral à Coordenação do setor; registrar e encaminhar às instâncias legais os casos de assédio moral sofrido pela equipe.

\section{b) Dimensão de Mobilização Social}

Investimento em informação sobre doação-transplante para grupos sociais em diferentes contextos: universidades/faculdades, escolas de nível médio/ profissionalizante, igrejas, festivais e encontros religiosos/estudantis, ações sociais e circo. Essa estratégia contribuiu frente às questões culturais que envolvem o processo doação-transplante;

Elaboração de cartilha sobre doação e transplante de órgãos à comunidade e folders aos familiares de pacientes em ME;

Mobilização junto à Assessoria de Comunicação (ASCOM) e ao Núcleo de Vigilância Epidemiológica, com confecção de materiais para treinamentos e campanhas;

Criada a "CIHDOTT Itinerante" para divulgar o processo doação-transplante no contexto comunitário, com realização das atividades descritas na figura 1 , segundo Lima; ${ }^{12}$
Divulgação/mobilização para participação na Campanha Estadual de Doação de Órgãos em Escolas, Faculdades, Universidades, Serviço Social do Comércio (SESC), grupos de ciclistas e motociclistas;

Mobilização nas redes sociais, emissoras de TV, rádio e jornais locais para a Campanha Estadual de Doação de Órgãos;

Confecção de painel de bonecos em MDF, com arte da Campanha Estadual de Doação de Órgãos. Nessa estrutura, as pessoas posicionavam-se para foto, cuja face completava a estrutura do boneco e demostravam apoio à doação de órgãos;

Sessão "Projeto Direito e Sétima Arte" vinculada a uma Faculdade sob ação integrada das Coordenações dos Cursos de Direito e Enfermagem. Nessa oportunidade, os estudantes discutiram e construíram argumentos sobre Doação de Órgãos;

Ação no Restaurante Universitário, com apoio dos alunos de Relações Públicas e da ASCOM, com distribuição de folders, painéis de fotos para declarar-se doador e convite para curtir fanpage da campanha em tempo real;

Ação de mobilização social em via pública do município de São Luís - Maranhão, com a utilização de painéis humanos e coreografias com frases sobre doação, distribuições de brindes e folders;

Realização de palestras sobre Doação de Órgãos em escolas e SESC;

Ações educativas nos shoppings do município de São Luís;

Entrevistas em rádios e jornais locais sobre doação e transplante de órgãos;

Campanha Estadual de Doação de Órgãos em 2014, 2015 e 2016, como produto de densas articulações e parcerias com instituições da saúde, educação e sociedade civil foram as maiores campanhas já realizadas no Maranhão;

Ação "Irmãos de Sangue" em parceria com o Hemocentro do Maranhão e a Igreja Católica sobre doação e transplante de órgãos;

Palestra "Tornando-se Doador" na Semana Nacional de Ciência e Tecnologia 2014/ V Mostra Científica do Maranhão, promovida pela FAPEMA, com participação da CNCDO e BO;

Participação da CIHDOTT e BO no Halleluya (evento religioso) com divulgação/ esclarecimentos sobre doação e transplante de órgãos;

Palestra sobre doação de órgãos na XX Assembleia Arquidiocesana de São Luís - Encontro de Casais, no Instituto Federal de Educação; 
Das fronteiras institucionais à mobilização social: intervenções no processo de doação-transplante

Figura 1: Atividades desenvolvidas pela CIHDOTT Itinerante de 2013 a 2016, São Luís-MA

\begin{tabular}{|c|c|c|c|}
\hline Ação & Local & Público alvo & Parcerias \\
\hline Encontro Acadêmico & UFMA & Alunos da área de saúde & Liga de Tanatologia \\
\hline Ação de sensibilização & Shoppings & Sociedade & $\begin{array}{l}\text { Movimento Escoteiro e } \\
\text { Hemocentro do Maranhão }\end{array}$ \\
\hline Ação de sensibilização & Via pública & Sociedade & Grupo “O Circo Tá na Rua” \\
\hline Roda educativa & Escola & Alunos de Enfermagem & CNCDO/ CIHDOTT/ BO \\
\hline Aula "Tornado-se Doador de Órgãos e Tecidos" & UFMA & Alunos de Enfermagem & Liga de Tanatologia \\
\hline $\begin{array}{l}\text { Encontro Regional Norte Nordeste de Nutrição } \\
\text { (XVI Erenut) }\end{array}$ & UFMA & Alunos de Nutrição & CIHDOTT \\
\hline $\begin{array}{l}\text { Palestra “Declare-se Doador de Órgãos e } \\
\text { Tecidos" }\end{array}$ & UNICEUMA & Alunos de Enfermagem & CIHDOTT \\
\hline Palestra "Doação de Órgãos" & Escola Ana Neri & Alunos de Enfermagem & CIHDOTT/ BO \\
\hline Jornada de Ética e Bioética & Hotel Luzeiro & Alunos de Direito & UNDB \\
\hline Ação Social & Igreja Nossa Senhora da Penha & Comunidade do Anjo da Guarda & CNCDO/ CIHDOTT \\
\hline Aula “Morte Encefálica” & Faculdade Pitágoras & Alunos de Enfermagem & CIHDOTT \\
\hline Projeto VER-SUS “Roda de conversa" & Hotel Premier & Alunos da área da saúde & CIHDOTT \\
\hline Aula “Doação de Órgãos" & UFMA & Alunos de Enfermagem & Liga de Tanatologia \\
\hline Aula "Transplante de órgãos" & CEUMA & Alunos de Medicina & Liga de Emergências \\
\hline $\begin{array}{l}\text { I Jornada de Enfermagem da Rede Estadual de } \\
\text { Saúde }\end{array}$ & Hospital Nina Rodrigues & Funcionários & Secretaria Estadual de Saúde \\
\hline Aula “Doação de Órgãos" & UFMA & Alunos de Enfermagem & CIHDOTT \\
\hline
\end{tabular}

Fonte: Lima ${ }^{12}$

Ações educativas sobre doação de órgãos nos terminais de Transportes Coletivos - São Luís;

Membros da CIHDOTT, CNCDO e BO realizaram mobilização social nas praias de São Luís no Reveillon de 2014, com distribuição de panfletos sobre doação de órgãos;

Todas as intervenções descritas acima produziram resultados exultantes no processo de doação e transplante e órgãos no estado do Maranhão, como podem ser observados nas tabelas 1 e 2, segundo dados da Secretaria da Saúde do Estado do Maranhão. ${ }^{13}$

Foram limitações da pesquisa o não investimento nas instituições que não compareceram às atividades programadas.

\section{DISCUSSÃO}

Para Arcanjo, Oliveira e Silva, a CIHDOTT e a CNCDO devem avaliar a capacidade da instituição em detectar potenciais doadores, considerando as características institucionais, suas competências de atendimento para captação de doadores e realização de transplantes, ${ }^{13}$ assim como, definir os parâmetros que deverão ser adotados no acompanhamento das metas de contratualização, definidas segundo a quantidade e qualidade das captações e transplantes determinadas pela Portaria $\mathrm{n}^{\circ}$. 1752/GM/MS, encaminhando ao gestor local os indicadores de desempenho estabelecidos para o hospital, adotando medidas para aperfeiçoar a captação de órgãos e tecidos e estabelecendo metas de atuação com prazo definido. ${ }^{14}$ 
Tabela 1: Notificação de morte encefálica segundo a CNCDO-MA de 2013 à 2016, São Luís - MA

\begin{tabular}{|c|c|c|c|c|c|c|c|c|}
\hline \multirow{2}{*}{ Ocorrências } & \multicolumn{2}{|c|}{2013} & \multicolumn{2}{|c|}{2014} & \multicolumn{2}{|c|}{2015} & \multicolumn{2}{|c|}{2016} \\
\hline & $\mathrm{n}$ & $\bar{x}$ & $\mathrm{n}$ & $\bar{x}$ & $\mathrm{n}$ & $\bar{x}$ & $\mathrm{n}$ & $\bar{x}$ \\
\hline Número de notificações de ME & 85 & 7,0 & 90 & 7,5 & 137 & 11,41 & 210 & 17,5 \\
\hline Número de protocolos de ME fechados & 21 & 1,75 & 33 & 2,75 & 91 & 7,58 & 87 & 7,25 \\
\hline
\end{tabular}

Fonte: Secretaria da Saúde do Estado do Maranhão ${ }^{13}$

Tabela 2: Total de transplantes de 2013 à 2016 de acordo com a origem dos doadores, São Luís-MA

\begin{tabular}{|c|c|c|c|c|c|c|c|c|}
\hline \multirow{2}{*}{ Transplantes de córnea } & \multicolumn{2}{|c|}{2013} & \multicolumn{2}{|c|}{2014} & \multicolumn{2}{|c|}{2015} & \multicolumn{2}{|c|}{2016} \\
\hline & $\mathrm{n}$ & $\bar{x}$ & $\mathrm{n}$ & $\bar{x}$ & $\mathrm{n}$ & $\bar{x}$ & $\mathrm{n}$ & $\bar{x}$ \\
\hline Doadores do Maranhão & 13 & 1,16 & 26 & 2,16 & 86 & 7,16 & 109 & 9,08 \\
\hline Doadores de outros estados & 106 & 9,58 & 81 & 6,75 & 58 & 4,83 & 69 & 5,75 \\
\hline Total & 119 & 10,75 & 107 & 8,91 & 144 & 12,0 & 178 & 14,83 \\
\hline \multirow{2}{*}{ Transplantes de Rim } & \multicolumn{2}{|c|}{2013} & \multicolumn{2}{|c|}{2014} & \multicolumn{2}{|c|}{2015} & \multicolumn{2}{|c|}{2016} \\
\hline & $\mathrm{n}$ & $\bar{x}$ & $\mathrm{n}$ & $\bar{x}$ & $\mathrm{n}$ & $\bar{x}$ & $n$ & $\bar{x}$ \\
\hline Doadores do Maranhão & 13 & 1,08 & 26 & 2,16 & 38 & 3,16 & 29 & 2,41 \\
\hline Doadores de outros estados & 16 & 1,33 & 08 & 0,66 & 23 & 1,91 & 04 & 0,3 \\
\hline Total & 29 & 2,41 & 32 & 2,66 & 61 & 5,08 & 33 & 0,71 \\
\hline
\end{tabular}

Fonte: Secretaria da Saúde do Estado do Maranhão ${ }^{13}$

O coordenador hospitalar de transplante é o profissional que executa a logística do processo de doação e transplante em seu âmbito e elabora programas educacionais direcionados aos profissionais de saúde e à sociedade, além de auxiliar a direção do hospital nos assuntos que envolvem o processo de doaçãotransplante. $^{2}$

A efetivação da doação é um processo complexo, em que é necessário que o médico identifique o potencial doador e que a família aceite a doação. Porém, se os profissionais de saúde e a família envolvidos nesse processo não forem educados sobre o tema, tornase mais difícil a decisão familiar nesse momento. A sociedade deve ser informada pelos profissionais de saúde sobre o conceito de $\mathrm{ME}$, como ocorre com a distribuição de órgãos, que o comércio não é permitido, que não há religião contrária à doação e que o corpo não fica deformado, entre outras dúvidas. ${ }^{2}$

O desconhecimento de como tornar-se doador e da definição de $M E$, além dos familiares não saberem a vontade do falecido são agravantes para a decisão contrária à doação, mas são questões que podem ser superadas com informações apropriadas de maneira clara e precisa, tirando dúvidas e superando medos comuns. A pessoa esclarecida pode promover conversa com familiares e amigos, colocando sua posição em relação à doação, ocasionando um dos fundamentais mecanismos de aceitação das doações. ${ }^{2}$

De maneira geral, a sociedade é informada por meio de comunicação de massa, tais como: televisão, rádio e jornais, que funcionam como importantes fontes de informações para assuntos de saúde, influenciando no comportamento, na prática profissional e em política nessa área. Campanhas e colaboração de artistas também podem influenciar os indivíduos a discutirem sobre o assunto. ${ }^{2}$

Entretanto, estudos demonstram que apesar da televisão ser um meio de comunicação de alto impacto para transmissão de informações, sua influência não é tão favorável para doação quanto a divulgada com base individual e em encontros específicos, como campanhas, profissionais de saúde, amigos ou familiares. ${ }^{2}$

É fundamental a educação para profissionais e estudantes da área da saúde, por serem formadores 
de opinião, além do importante papel do atendimento do profissional de saúde na satisfação dos familiares quanto à qualidade do tratamento dispensado ao falecido e à comunicação da família com a equipe. ${ }^{2}$

Outra dificuldade que a doação de órgãos enfrenta é a ignorância da sociedade sobre o assunto, e por isso, a conscientização sobre o tema é importante, principalmente com o esclarecimento do complexo processo do ato de doar, desmistificando-o e tornando-o um ato generoso, consciente e solidário com o próximo, iniciativa que cabe aos profissionais de saúde e às Organizações da Sociedade Civil (OSC) que são favoráveis à causa. ${ }^{2}$

\section{CONCLUSÃO}

Esta investigação provocou e promoveu avanços quantitativos e qualitativos, com envolvimento nas dimensões institucionais, técnicas, operacionais e burocráticas. Foram produtos desta investigação as seguintes transformações no processo de doação e transplante do estado do Maranhão: aumento do número de notificações de óbitos e mortes encefálicas; maior número de hospitais concluindo o protocolo de ME; agilidade no tempo de realização do protocolo de ME; implantação de novas CIHDOTTs; aumento do número de profissionais na área de captação de órgãos; viabilidade para realização do exame complementar do protocolo de ME e aumento de doações e transplantes.

Os resultados da pesquisa evidenciaram que a participação ativa dos atores ao somar seus potenciais conseguiu transformar $o$ cenário do diagnóstico situacional do estado do Maranhão, aumentando o número de doações e de transplantes de órgãos e tecidos. Das intervenções planejadas no decorrer da pesquisa na dimensão institucional e técnicaprofissional, foram destaques a capacitação operacional dos profissionais como suporte ao processo doação e transplante e a qualificação no manejo terapêutico do potencial doador.

\section{ABSTRACT}

Purpose: From the active participation of the agents involved in the organ donation, retrieval and transplantation process, to create innovative measures aiming to increase the notifications, donations and transplants in the State of Maranhão. Methods: research by qualitative approach following the Convergent Healthcare Research model. Data were collected by the following procedures: conversation meetings, focus groups, individual meetings, and daily practices involving the organ donation process. Results: Results were presented in three dimensions: institutional, technical-professional, and social mobilization. Conclusion: Interventions were defined as innovative measures represented by the increase of brain death notifications.

Keywords: Organ Donation; Tissue Donation; Transplantation.

\section{REFERENCES}

1. Andersen $H$, Borre M, Jakobsen J, Heden Andersen $P$, Vilstrup $H$. Decreased muscle strength in patients with alcoholic liver cirrhosis in relation to nutritional status, alcohol abstinence, liver function and neuropathy. Hepatology. 1998;27(5):1200-7.

2. Rodes J, Navasa M. Liver transplantation and quality of life. Can J Gastroenterol. 2000;14(8):693-9.

3. Schlenk EA, Erlen JA, Dunbar-Jacob J, McDowell J, Engberg $S$, Sereika SM, et al. Health-related quality of life in chronic disorders: a comparison across studies using the MOS SF-36. Qual Life Res. 1998;7(1):57-65.

4. Pugh RNH, Muray Lyon IM, Dawson JL, Pietroni MC, Williams $\mathrm{R}$. Transaction of the oesophagus for bleeding oesophageal varices. Br J Surg. 1973;60(8):646-9.

5. Boin IFSF, Leonardi MI, Udo EY, Pereira TS, Stucchi RSB, Leonardi LS. The application of MELD score in patients submitted to liver transplantation: a retrospective analysis of survival and the predictive factors in the short and long term. ArqGastroenterol. 2008;45(4):275-83.
6. Malinchoc M, Kamath PS, Gordon FD, Peine CJ, Rank J, ter Borg PC. A model to predict survival in patients undergoing transjugular intrahepatic portosystemic shunts. Hepatology. 2000;31(4):864-71.

7. Brazil, Republic Presidency, Ministry of Health. Ministerial Directive no. 1.160 of May 29th, 2006. Criteria for the distribution of liver from deceased donors for transplantation. Union's Official Journal, Brasília (DF) 2006 May 31 no.103.

8. Wiesinger GF, Quittan M, Zimmermmann K,Nuhr M, Wichlas $M$, Bodingbauer $M$, et al. Physical performance and healthrelated quality of life in men on liver transplantation waiting list. J Rehabil Med. 2001;33(6):260-5.

9. Alameri HF, Sanai FM, Dukhayil M, Azzam NA, Al-Swat $K A$, Hersi $A S$, et al. Six minute walk test to assess functional capacity in chronic liver disease patients. World $\mathrm{J}$ Gastroenterol. 2007;13(29):3996-4001.

10. Saab S, Ibrahim AB, Shpaner A,Younossi ZM, Lee C, Durazo $F$, et al. MELD fails to measure quality of life in liver transplant candidates. Liver Transpl. 2005;11(2):218-23 